Calleja, M.A.I., \& Ruiz, I. (2013). La experiencia de coordinación en el Grado de Maestro de Educación Infantil. Revista Electrónica Interuniversitaria de Formación del Profesorado, 16(1), 15 -24.

\title{
La experiencia de coordinación en el Grado de Maestro de Educación Infantil
}

Ma A. Inmaculada Calleja González, Inés Ruiz Requies

Universidad de Valladolid

\section{Resumen}

El objeto de este trabajo fue dar a conocer la percepción detectada, sobre la implantación del Grado de Maestro en Educación Infantil adaptado al EEES y proponer sugerencias de mejora. Se realizó a través de un análisis DAFO, al profesorado y al alumnado de la Facultad de Educación y Trabajo Social de la Universidad de Valladolid. Los datos procedieron de diferentes evaluaciones llevadas a cabo por el Comité de Título durante los primeros cursos académicos de estos estudios, dando respuesta a una de las competencias asignadas: proponer mejoras en el programa formativo y en los servicios prestados en la titulación.

Palabras clave

Coordinación; EEES; Grado de Educación Infantil.

\section{The experience of coordination in the Teaching Degree in pre- primary Education}

\begin{abstract}
The objective of this work was to disseminate the perception detected about the implementation of the Teaching Degree in pre-primary Education adapted to the EHEA and propose suggestions for improvement. It was performed through a SWOT analysis, to the teaching staff and students of the Faculty of Education and Social Work at the University of Valladolid. The data came from different assessments carried out by the Committee of Title during the first years of these studies, responding to one of the assigned competencies: To propose improvements in the educational program and in the services provided in the degree.
\end{abstract}

\section{Key words}

Coordination; EHEA; Teaching Degree in pre-primary Education. 


\section{Introducción}

Tras la publicación de la Ley Orgánica 4/2007, en la que se modificó la Ley Orgánica de Universidades 6/2001 y el Real Decreto 1393/2007, ordenando las enseñanzas universitarias, la Universidad de Valladolid ha transformado los antiguos Planes de Magisterio en los nuevos Grados de Educación. Para garantizar el adecuado funcionamiento de los títulos se constituyeron diferentes Comisiones de Calidad, entre ellas, la Comisión de Calidad de Título. Entre las competencias a ella asignadas, cobran cierta relevancia aquellas encardas de seguir el cumplimiento de los planes anuales de mejora de la titulación y proponer mejoras sobre el programa formativo y de los servicios prestados. En este trabajo, se describen las actuaciones concretas que la Facultad de Educación y Trabajo Social de la Universidad de Valladolid, a través del Comité del Título, ha llevado a cabo para su adaptación al Espacio Europeo de Educación Superior (EEES) y plantea una serie de reflexiones y posibles mejoras, aportadas por el profesorado y por el alumnado en los diferentes análisis DAFO aplicados durante los dos primeros cursos académicos de la implantación del Grado de Infantil.

\section{Lo que ha supuesto la implantación de los Grados según el EEES}

La modificación de la Ley Orgánica de Universidades 6/2001, de 21 de diciembre ha permitido sentar las bases para llevar a cabo una profunda modernización de la Universidad española. El Título VI de la Ley establece una nueva estructuración de las enseñanzas y de los títulos universitarios oficiales lo que ha permitido reorientar el proceso de convergencia de nuestras enseñanzas universitarias con los principios de la construcción del EEES. En la posterior publicación del Real Decreto 1393/2007, de 29 de octubre, se establecía la ordenación de las enseñanzas universitarias, profundizando en la concepción y expresión de la autonomía universitaria. Gracias a esta autonomía, las universidades respetando las reglas establecidas, han propuesto aquellas enseñanzas y títulos que han considerado más adecuados impartir, sin haberse visto sujetas a la existencia de un catálogo previo establecido por el Gobierno.

Otro aspecto importante que permitía esta ley, era la flexibilización en la organización de las enseñanzas universitarias, lo cual ha favorecido y propiciado un mejor aprovechamiento de las fortalezas de cada universidad, incorporar las innovaciones realizadas y promover una diversidad curricular al objeto de dar respuesta a las demandas de una sociedad plural y en transformación continua.

Unido a esto se introdujo un cambio en las metodologías docentes, éstas debían centrarse en los procesos de aprendizaje del estudiante e inculcarles que el aprendizaje no finaliza con la adquisición de un determinado Grado o Máster sino que debe de ser constante y continuo a lo largo del ciclo vital.

Ahora bien, en el diseño y aplicación de un determinado título, es preciso ir más allá de la descripción de los contenidos formativos, se requiere incorporar nuevos elementos: justificación, objetivos, admisión de estudiantes, contenidos, planificación, recursos, desarrollo de competencias, resultados previstos y sistema de garantía de calidad. Uno de los conceptos claves que se incorpora es la adquisición y desarrollo de competencias en el alumnado. Entendiendo por competencias como la capacidad aprendida para realizar de manera adecuada una tarea, función o rol relacionada con el ámbito particular de trabajo que integra conocimientos, habilidades y actitudes (Carreras \& Perrenoud, 2005) y donde el aprendizaje por competencias no se orienta al mero desempeño inmediato de habilidades, sino que contempla la educación integral del estudiante (Torrego y Ruiz, 2011).

No obstante, para que un título permita el desarrollo de las competencias debe de hacer hincapié en los métodos de aprendizaje de dichas competencias y en los procedimientos para evaluar su desarrollo y adquisición. Unos métodos de aprendizaje que no sean meramente expositivos y reproductivos sino que sean métodos centrados en la educación de la persona que tal y como define Ander-Egg serían: "medios y procedimientos que, utilizados en situaciones de grupo, sirve para facilitar y estimular la acción y funcionamiento del grupo, de cara a alcanzar sus propios objetivos" (1986, p. 17). Tampoco podemos olvidar la necesidad de evaluar el proceso de adquisición y 
desarrollo de competencias, el cual es complejo y requiere una investigación en profundidad. Posiblemente, el proceso de evaluación debería permitir una evaluación tridimensional (Espinosa, Jiménez, Olabe y Basogain, 2006) entre las competencias a desarrollar por parte del alumnado, las tareas a llevar a cabo durante el curso para desarrollar dichas competencias, y los criterios de evaluación que se utilicen para la evaluación de las mismas. Así como una evaluación desde la perspectiva integral y global de la persona y del aprendizaje (Pérez Gómez, 2007)

Otro concepto nuevo que introduce la Ley es el crédito europeo, las anteriores horas lectivas se trasforman en créditos ECTS (European Credit Transfer System), una nueva medida que debe reflejar los resultados de aprendizaje y el volumen de trabajo realizado por el estudiante para alcanzar los objetivos establecidos en el plan de estudios, siendo la motivación y el esfuerzo del estudiante para aprender los ejes que deben guiar este aprendizaje.

El Real Decreto 1393/2007, en su exposición de motivos, también alude a los Sistemas de Garantía de la Calidad como parte imprescindible de los nuevos Planes de Estudios. Con estos sistemas de calidad se pretende garantizar el adecuado funcionamiento de los títulos, crear confianza en los procesos de evaluación y acreditación, ya que permiten la supervisión efectiva de la enseñanza, e informar a la sociedad sobre la calidad de la misma. Es aquí donde los Comités de Título y las tareas de Coordinación tienen su razón de ser.

\section{Actuaciones concretas en la Universidad de Valladolid.}

Para poder implantar el EEES, la Universidad de Valladolid ha llevado a cabo una serie de medidas concretas de actuación. En relación a la metodología docente se definió un nuevo patrón de docencia en el cual las asignaturas de un determinado Plan de Estudios se podían dividir en clases teóricas, clases prácticas, seminarios, etc. y combinar distintos métodos de enseñanza en cada una de ellas. Siguiendo las orientaciones de Guilarte, Marbán y Miranda (2008), en función del modelo de clase en la que se fuera a intervenir, se podía elegir la metodología más idónea entre las que destacamos: la lección magistral, el estudio de casos, el aprendizaje cooperativo, etc. para la modalidad clases teóricas y expositivas; la resolución de problemas, el aprendizaje basado en problemas, etc. para las clases prácticas y el estudio de casos, la resolución de problemas, etc. para los seminarios. Esto no quiere decir que sean éstos los métodos únicos, ya que el actual sistema universitario requiere de una gran variedad de métodos de enseñanza en función del tipo de asignatura, el número de alumnos, el espacio con el que se disponga y la creatividad del docente.

Ahora bien, estas propuestas debían tender hacia una renovación metodológica que repercutiera tanto en la mejora del aprendizaje del alumnado como en el incremento del nivel de satisfacción y motivación de profesores y estudiantes. Además deberían conllevar avances claros hacia un nuevo estilo de trabajo del profesorado en el que se combinara satisfactoriamente la formación básica de los estudiantes, con una mayor aproximación al ejercicio profesional para el que se les está preparando (MEC, 2006, p. 84).

Por otro lado, los nuevos créditos ECTS han supuesto una modificación tanto en el patrón de docencia, como en los horarios y en la carga de trabajo del profesorado y del alumnado. Éstos, además de las horas presenciales, que puede variar en los diferentes grados entre el $30040 \%$ de presencialidad, también deben dedicar un número importante a las horas de trabajo individual en cada asignatura.

Por lo que se refiere al título objeto de análisis, la Universidad de Valladolid, amparada en las nuevas medidas de la Ley, diseñó, elaboró y propuso el Plan de Estudios del Título de Grado Maestro/a en Educación Infantil. Un plan único que se ha llevando a cabo en los cuatro centros adscritos a ella: Escuela Universitaria de Educación de Palencia; Escuela Universitaria de Magisterio de Segovia, Escuela Universitaria de Educación de Soria y Facultad de Educación y Trabajo Social de Valladolid y que de forma pionera se empezó a aplicar durante el curso académico 2009-10, un curso académico anterior a la fecha obligatoria para adaptar todos los títulos al EEES.

Inicialmente, en el Consejo de Gobierno de 24 de Julio de 2008, se aprobó el Sistema de Garantía Interno de Calidad de los Títulos Oficiales de Grado de la Universidad de Valladolid. En dicho Sistema, 
se especifican diferentes tipos de comisiones como responsables del sistema de garantía de la calidad del plan de estudios: Comisión de Calidad de la Universidad de Valladolid; Comisión de Garantía de Calidad del Centro; Comisión de Título y en aquellos casos que se imparta la misma titulación en varios centros la Comisión de Garantía de Calidad Intercentros.

\section{La coordinación en el Grado de Maestro de Educación Infantil en la Facultad de Educación y Trabajo Social de la UVa.}

Durante los cursos académicos 2009-10; 2010-11 y 2011-12 se ha impartido los tres primeros cursos del Grado de Maestro en Educación Infantil en los cuatro campus anteriormente mencionados. Para llevar a cabo una correcta implantación ha sido necesaria una estrecha y profunda coordinación entre todos los implicados a distintas bandas. Por un lado, una coordinación del Título, en este caso, era la Comisión del Título de Grado en Educación Infantil, la que ha intentado dar respuestas a las peculiaridades y problemáticas específicas del plan de estudios. Por otro, ha sido necesario realizar una estrecha coordinación entre todos los títulos implantados en un mismo centro, para armonizar la puesta en marcha de los diferentes Grados. En concreto en la Facultad de Educación y Trabajo Social de Valladolid durante el curso 2009-2010 se implantaron los siguientes grados: Grado en Educación Primaria, Grado en Educación Infantil y Grado en Educación Social y en el curso 2010-12 el título de Trabajo Social, los Coordinadores de estos títulos formaban parte de la Comisión de Garantía de Calidad del Centro. Y por último, una coordinación entre los cuatro centros del campus para poder llevar a cabo los planes implantados respetando criterios comunes (Comisión de Coordinación Intercentros).

Durante el primer año de aplicación, al ser una experiencia piloto, se llevaron a cabo numerosas reuniones en cada una de las comisiones. Era necesario acomodar y adecuar la nueva normativa, introducir sugerencias de modificación en algún punto, proponer nuevas normativas en función de las necesidades y demandas detectadas, etc. lo que propició una fuerte cohesión entre los cuatros campus.

Sin embargo, las condiciones de cada campus, sus peculiaridades, los grados concretos que se han impartidos en cada centro, el número de alumnos, el número de grupos, el número de menciones, han diferido entre sí. Por eso, con el devenir de estos años y la implantación de las nuevas ofertas educativas: Curso de adaptación al Grado, incorporación de las nuevas Menciones en los diferentes campus, etc. ha propiciado una cierta autonomía en cada uno de los centros disminuyendo la cohesión inicial.

Respecto a la Comisión de Calidad de Título del Grado en Educación Infantil de la Facultad de Educación y Trabajo Social de Valladolid, fue nombrada por la Junta del Centro y ha estado compuesta por:

a) La coordinadora de titulación.

b) 10 profesores pertenecientes a diferentes departamentos implicados en la titulación.

c) Un representante del Personal de Administración y Servicios del Centro.

d) Un estudiante con al menos el $50 \%$ de los créditos aprobados.

Las competencias asignadas a dicha comisión por el Sistema de Garantía Interno de Calidad de los Títulos Oficiales de Grado de la Universidad de Valladolid nos han obligado a realizar una intensa actividad para poder dar respuesta a muchas de las cuestiones suscitadas a los largo de este tiempo. Entre dichas competencias destacamos:

a) Seguir el cumplimiento de los planes anuales de mejora de la titulación.

b) Evaluar los resultados del Sistema de Garantía Interno de Calidad respecto a la titulación.

c) Proponer mejoras sobre el programa formativo y los servicios prestados.

d) Elevar propuestas al centro en materia de ordenación académica del título y sobre la asignación docente. 
e) Coordinar y planificar la metodología de enseñanza, los programas de actividades y la evaluación de aprendizajes del título.

f) Realizar el análisis de las causas y buscar soluciones a las incidencias, reclamaciones y sugerencias que en el ámbito del título se han planteado.

Además de estas competencias, dicha comisión de calidad ha tenido que asumir otras no menos importantes, entre las que destacamos:

a) Diseñar y organizar el Practicum del Grado de Educación Infantil.

b) Diseñar y organizar el Curso de Adaptación al Grado y los correspondientes Trabajos de Fin de Grado: Guía docente, elección de tutores, líneas de intervención, tribunales de evaluación, etc.

Gran parte de las actividades e intervenciones realizadas, así como el resultado de las evaluaciones llevadas a cabo por la Universidad, tanto al alumnado como al profesorado y al Personal de Administración y Servicios (PAS), se hallan reflejados en los Autoinformes de Seguimiento del Título de Infantil remitidos al ACSUCYL (Agencia para la Calidad del Sistema Universitario de Castilla y León). Ahora bien, durante estos años también se han realizado, desde el Comité de Título del Grado en Educación Infantil, una serie de evaluaciones tanto al alumnado, profesorado y PAS y parte de esos resultados nos gustaría destacar aquí.

Uno de los objetivos propuestos en estas evaluaciones era recabar información acerca de la percepción que tenían los agentes implicados en este proceso respecto a la adaptación de las enseñanzas al EEES. Percepciones sobre los cambios introducidos, tanto en las modalidades docentes (clase magistrales con el gran grupo, prácticas de aula con la mitad del gran grupo y seminarios con la cuarta parte del gran grupo), en las estrategias docentes, la aplicación de los Créditos ECTS, la organización de horarios, como la dedicación del alumno al trabajo personal, etc.

Durante los dos primeros años de implantación del Grado al finalizar cada uno de los cuatrimestres se aplicó un análisis DAFO tanto al alumnado como al profesorado para tener una información de la realidad percibida y en función de los datos introducir, en la medida de lo posible, las adaptaciones o los cambios pertinentes.

De esta forma, pudimos detectar una serie de fortalezas y debilidades, por un lado, referidas al nuevo modelo educativo adaptado al EEES y por otro, a la aplicación concreta que se hacía en cada una de las asignaturas de los cursos, así como una serie de sugerencias de mejora que se intentaron llevar a cabo, en la medida de lo posible.

Entre los resultados obtenidos durante estos cursos destacamos las respuestas más significativas y repetidas referidas a la percepción por parte de profesorado respecto a la adaptación del Título del Grado de E.I. al EEES (Tabla 1), sobre la aplicación del EEES a cada asignatura (Tabla 2) y del alumnado durante estos años (Tabla 3).

Tabla 1. Percepción del profesorado respecto a la adaptación del Título de Grado en Educación Infantil al Espacio Europeo de Educación Superior

\begin{tabular}{|l|l|}
\hline Fortalezas & Debilidades \\
\hline $\begin{array}{l}\text { Hacer una reflexión previa favorece la } \\
\text { organización y planificación de las asignaturas. }\end{array}$ & $\begin{array}{l}\text { La planificación a largo plazo limita las posibles } \\
\text { modificaciones que surgen a lo largo del curso. }\end{array}$ \\
$\begin{array}{l}\text { Se favorece el desarrollo de competencias que } \\
\text { el anterior modelo no contemplaba de forma } \\
\text { explícita. }\end{array}$ & $\begin{array}{l}\text { La escasa formación previa del alumnado de } \\
\text { primer curso dificulta el desarrollo de } \\
\text { competencias generales y específicas. La escasa } \\
\text { competencia para la lectura, síntesis y y } \\
\text { comprensión del material bibliográfico científico } \\
\text { es un freno para el desarrollo de otras } \\
\text { competencias. } \\
\text { En primer curso, al desconocer el alumnado } \\
\text { totalmente la asignatura, resulta difícil realizar }\end{array}$ \\
\hline
\end{tabular}


Las prácticas programadas pueden ayudar a la interiorización de los contenidos teóricos, sobre todo en los cursos superiores.

La organización y planificación del trabajo puede ayudar al alumno a responsabilizarse de su propio proceso de aprendizaje.

El alumno puede tomar un mayor contacto con la realidad.

La participación en los seminarios permite una profundización de los contenidos.

El programa Orienta, iniciado el curso 2009-10 se consideró muy útil tanto para profesores como para el alumnado trabajos prácticos con cierto rigor.

El exceso de prácticas, supone un gran trabajo y no siempre se tienen interiorizados los contenidos previos imprescindibles para llevar a cabo con éxito las prácticas.

La escasa implicación, por parte de cierto alumnado, para cumplir la planificación ha dificultado el trabajo en grupo y el rendimiento individual.

La rigidez en el horario ha limitado un contacto más directo con las instituciones externas a la Universidad.

Dificultades en la implicación de todo el alumnado en los seminarios ya que no está habituado a realizar un trabajo individual en profundidad.

La escasez de fondos y de reconocimiento por las instituciones hacia este tipo de servició, contribuyó a que se extinguiera.

Tabla 2. Percepción del profesorado sobre la aplicación del EEES a cada asignatura.

\begin{tabular}{|c|c|}
\hline Fortalezas & Debilidades \\
\hline $\begin{array}{l}\text { Las experiencias de aprendizaje tanto en gran } \\
\text { grupo como en prácticas han sido muy } \\
\text { satisfactorias, con dificultades en los seminarios. }\end{array}$ & $\begin{array}{l}\text { Es preciso dotar de entidad propia a los } \\
\text { seminarios, no todos entendemos lo mismo y al } \\
\text { alumnado en ocasiones le crea confusión. }\end{array}$ \\
\hline $\begin{array}{l}\text { El trabajo en grupo cuando se hace bien es muy } \\
\text { enriquecedor y favorece el conocimiento. }\end{array}$ & $\begin{array}{l}\text { Dificultades en ciertos trabajos en grupo, más } \\
\text { que un trabajo colaborativo es la suma de } \\
\text { trabajos individuales, falta debate y acuerdos. }\end{array}$ \\
\hline $\begin{array}{l}\text { La disminución de la ratio ha permitido un } \\
\text { aumento de la interacción con el alumnado }\end{array}$ & $\begin{array}{l}\text { La ubicación de ciertas asignaturas en los } \\
\text { semestres y curso no se considera la más } \\
\text { adecuada: Fundamentos de la Atención } \\
\text { Temprana (primer semestre), Infancia y Hábitos } \\
\text { de Vida Saludable, etc. Impartidos en el primer } \\
\text { semestre de primer curso deberían impartirse en } \\
\text { cursos posteriores cuando el alumnado haya } \\
\text { adquirido unos conocimientos previos y } \\
\text { desarrollado ciertas competencias básicas. }\end{array}$ \\
\hline $\begin{array}{l}\text { Las diferentes modalidades docentes exigen una } \\
\text { utilización de espacios adaptados para ello. }\end{array}$ & $\begin{array}{l}\text { Los espacios de los centros resultan } \\
\text { insuficientes para poder albergar el trabajo } \\
\text { individual o en grupo. En las horas no } \\
\text { presenciales de éstos, en función de la } \\
\text { modalidad docente. }\end{array}$ \\
\hline
\end{tabular}


Tabla 3. Percepción del alumnado sobre la experiencia de implantación.

\begin{tabular}{|c|c|}
\hline Fortalezas & Debilidades \\
\hline $\begin{array}{l}\text { Las experiencias de aprendizaje tanto en gran } \\
\text { grupo como en prácticas han sido muy } \\
\text { satisfactorias, con dificultades en los seminarios. }\end{array}$ & $\begin{array}{l}\text { Es preciso dotar de entidad propia a los } \\
\text { seminarios, no todos entendemos lo mismo y al } \\
\text { alumnado en ocasiones le crea confusión. }\end{array}$ \\
\hline $\begin{array}{l}\text { El trabajo en grupo cuando se hace bien es muy } \\
\text { enriquecedor y favorece el conocimiento. }\end{array}$ & $\begin{array}{l}\text { Dificultades en ciertos trabajos en grupo, más } \\
\text { que un trabajo colaborativo es la suma de } \\
\text { trabajos individuales, falta debate y acuerdos. }\end{array}$ \\
\hline $\begin{array}{l}\text { La disminución de la ratio ha permitido un } \\
\text { aumento de la interacción con el alumnado }\end{array}$ & $\begin{array}{l}\text { La ubicación de ciertas asignaturas en los } \\
\text { semestres y curso no se considera la más } \\
\text { adecuada: Fundamentos de la Atención } \\
\text { Temprana (primer semestre), Infancia y Hábitos } \\
\text { de Vida Saludable, etc. Impartidos en el primer } \\
\text { semestre de primer curso deberían impartirse en } \\
\text { cursos posteriores cuando el alumnado haya } \\
\text { adquirido unos conocimientos previos y } \\
\text { desarrollado ciertas competencias básicas. }\end{array}$ \\
\hline $\begin{array}{l}\text { Las diferentes modalidades docentes exigen una } \\
\text { utilización de espacios adaptados para ello. }\end{array}$ & $\begin{array}{l}\text { Los espacios de los centros resultan } \\
\text { insuficientes para poder albergar el trabajo } \\
\text { individual o en grupo. En las horas no } \\
\text { presenciales de éstos, en función de la } \\
\text { modalidad docente. }\end{array}$ \\
\hline
\end{tabular}

\section{Propuestas de Mejora}

Entre las diferentes sugerencias de mejora destacamos las siguientes:

a) Reflexionar sobre la entidad didáctica de los seminarios.

b) Necesidad de una mayor coordinación entre los profesores que imparten asignaturas similares y sobre todo entre el profesorado de diferentes departamentos que imparten una misma materia.

c) Mejorar la coordinación entre el profesorado relativa a la entrega de trabajos y actividades en un mismo grupo.

d) Revisar la ubicación de ciertas materias a lo largo del Plan de Estudios.

e) Las asignaturas con más de seis créditos ECTS deberían distribuirse a lo largo del año y no ser semestrales.

f) Respetar las Guías Docentes publicadas al principio de curso ya que los cambios pueden afectar a la programación del resto de asignaturas.

g) Coordinación periódica entre el profesorado que imparte docencia en un mismo curso y plan de estudios para consensuar contenidos y que éstos no se repitan en distintas asignaturas.

h) Revisar los horarios para evitar los "tiempos muertos", en los que el alumnado no está dentro del aula y tiene que permanecer en el centro para trabajar de forma autónoma.

i) Fomentar la interdisciplinariedad entre asignaturas, por ejemplo aumentar la coordinación entre las distintas asignaturas con las TIC.

j) Mayor flexibilidad en el patrón de docencia para poder adaptarse a la metodología concreta que cada una de las asignaturas requiere.

k) Mayor flexibilidad en la organización de los grupos de prácticas o seminario teniendo en cuenta las sugerencias propuestas por el grupo. 
I) Generar dinámicas de evaluación más adaptadas a las nuevas metodologías docentes.

\section{Conclusiones}

Una vez analizados estos tres primeros años de implantación del Grado de Educación Infantil al EEES en la Facultad de Educación y Trabajo Social de la Universidad de Valladolid, consideramos que el esfuerzo realizado por todos los agentes implicados: Decanato, Comité de Título, Junta de Facultad, profesorado, alumnado y Personal de Administración y Servicios, ha sido intenso y continuado durante los tres cursos académicos a los que hemos hecho mención.

Un esfuerzo importante ya que al haber sido pioneros en la adaptación del título al nuevo sistema educativo, ha sido necesario dar respuesta a numerosas solicitudes (convalidaciones de asignaturas con otros títulos, con estudios de Formación Profesional, etc.), a un nuevo Practicum más exigente y con una normativa que anteriormente no existía y a nuevos requerimientos como los Curso de Adaptación al Grado o el Trabajo de Fin de Grado, totalmente nuevos en nuestra titulación. Todo ello, ha requerido una continúa demanda de normativa y legislación, inexistente hasta el momento, para poder llevar a cabo la gestión del título. Esta falta de normativa dificultó aún más el trabajo de coordinación ya que en sucesivas ocasiones se ha tenido que adaptar y modificar el trabajo realizado tras la publicación de las normativas.

Por otro lado, queremos destacar el esfuerzo continuado de todos al intentar aplicar con rigor las nuevas metodologías docentes, las cuales, nos han obligado a introducir modificaciones, redefiniciones y nuevos planteamientos en función de las sugerencias de mejora propuestas por los distintos sectores implicados. Los datos recogidos a través de los análisis DAFO, tanto del profesorado como del alumnado, han permitido cuidar la calidad educativa e introducir mejoras a lo largo de todo este proceso.

Ahora bien, llegados a este punto, con todo este bagaje de funcionamiento, no podemos dejar de hacernos ciertas preguntas ¿Y ahora qué? ¿Qué va a pasar tras los nuevos cambios introducidos por la crítica situación económica que padecemos? ¿Los ajustes aplicados al personal docente e investigador, el incremento en el número de asignaturas impartidas por el profesorado, el excesivo número de alumnos por aula, la dificultad para realizar seminarios con un número de alumnos más reducido, va a permitir llevar a cabo con rigor las diferentes metodologías ya puestas en práctica y, en cierta medida, consolidadas? Son muchos los interrogantes que podríamos plantear ante esta manifiesta sensación de pérdida que nos invade.

No obstante, no queremos finalizar esta reflexión sin poner en valor lo mucho que se ha conseguido durante estos tres años, el cambio de actitud por parte del profesorado hacia la nueva forma de enseñar, el esfuerzo de éste, por introducir nuevas metodologías docentes y coordinarse con sus compañeros y compañeras, por combinar teoría y práctica y por intentar acercar su práctica a la realidad. Deseamos que todos estos esfuerzos sigan siendo significativos ya que, pese a la situación en la que estamos inmersos, hoy por hoy sigue imperando la responsabilidad y la implicación de todos para alcanzar la adaptación definitiva de la titulación al Espacio Europeo de Educación Superior

\section{Bibliografía}

Ander Egg, E. (1986). Técnicas de reuniones de trabajo. Buenos Aires: Humanistas.

Carreras, J., \& Perrenoud, P. (2005). El debat sobre les competències a l'ensenyament universitari. Barceolona: Universitat de Barcelona.

Espinosa J.K., Jiménez, J., Olabe, M.A., \& Basogain, X. (2006). Innovación docente para el desarrollo de competencias en el EEES. Tecnologías aplicadas a la enseñanza de la Electrónica. Congreso Tecnologías Aplicadas a la Enseñanza de la Electrónica (TAEE 2006). Consulta 2 Septiembre 2007 de http://www.euitt.upm.es/taee06/papers/S4/p216.pdf

Guilarte, C., Marbán, J.M., \& Miranda, S. (2008). Principios básicos para el diseño de guías docentes de asignaturas en el marco del EEES así como la rigidez en el horario Castilla y León: 
Valladolid.

Recuperado

de

http://www.uva.es/uva/export/portal/com/bin/contenidos/gobiernoUVA/Vicerrectorados/Vic

erectoradoCalidadInnovacion/Innovacion_educativa/materiales/mat_prof/Guia_docente_asi gnatura/1215505533222_ppos_basicos_guias_docentes_eees_uva.pdf

MEC (2006). Propuestas para la renovación de las metodologías educativas. Recuperado de http://www.mec.es/educa/jsp/plantilla.jsp?area=ccuniv\&id=910

Pérez, A. (2007). La naturaleza de las competencias básicas y sus aplicaciones pedagógicas. Cuadernos de pedagogía de Cantabria (1).

Torrego, L., \& Ruiz Esteban, C. (2011). La coordinación docente en la implantación de los títulos de Grado. REIFOP, 14(4). Recuperado de http://www.aufop.com 


\section{Autores}

$M^{a}$ A. Inmaculada Calleja González

Profesora Titular de Escuela Universitaria del Departamento de Psicología. Imparto las asignaturas del área de Evolutiva y de la Educación en concreto: Psicología del Desarrollo, Psicología del Aprendizaje en Contextos Educativos, Evaluación y Diagnóstico. Mis líneas de investigaciones son: Evaluación e intervención en el desarrollo del Razonamiento Inductivo en niños de infantil y Primaria. Diagnostico e intervención en TDAH e Innovación educativa en Educación Superior

Inés Ruiz Requies

Profesora del Departamento de Pedagogía de la FEYTS. Actualmente imparto las asignaturas de Didáctica General y Currículo y Sistema Educativo en la Formación Inicial de maestros/as. He participado en la organización de numerosas actividades e investigaciones I+D y soy miembro del Grupo GSIC-EMIC. Mis líneas de investigación son el CSCL, la innovación educativa en Educación Superior y las TICs en los procesos de enseñanzaaprendizaje 